\title{
The Geometrical Frame Optimize Method to Evaluate and Research the Circumferentially Noniform Hole Group Position Error
}

\author{
Zhang Rong \\ Department of Mechanical Engineering, Dalian Vocational \& Technical College, Dalian, Liaoning, \\ 116037 ,China \\ Lzhch11993@sina.com
}

Keywords: Position error; Hole group; Optimize; Rotate vector

\begin{abstract}
How to evaluate the position errors of a hole group correctly is a key point in application and dissemination of position tolerance. In a coincident tolerance zone, a new concept, rotation of the theoretical center of the hole group, is raised here, by means of it, the geometrical reference frame of the hole group can be rotated. Therefore, in the graph of coincident tolerance zone, the geometrical reference frame can be both translated and rotated and the position errors of the hole group can be obtained using graphic method. Frame optimization method is intuitive and image geometric features of the principle proposed in view of the above problems in this paper, it is easy to analysis the parts, because it have a high precision and solving speed and can draw solution graphics visually. In this paper, a series of mathematical models are established and a microcomputer is used to process the data and draw the graphs, therefore, the calculating accuracy and speed are improved greatly.
\end{abstract}

\section{Introduction}

At present, the inspection hole position error in domestic and foreign usually adopt synthetic gauge inspection, synthetic gauge is a state of simulation assembly inspection method, which marked and position tolerance to adapt. For mass production, using synthetic gauge inspection which is a reasonable test method with its high efficiency, but it can't measure the position error of its exact figures. For single and small batch production of parts, using synthetic gauge inspection is expensive; The manufacturing error of synthetic gauge have great effect on the test results, and it have not a solution of how to assess the manufacturing error of synthetic gauge. If the position requires a high degree of accuracy, the comprehensive gauge even difficult to manufacture [1-3].

If using the graphic method in coordinate measurement device and measuring the coordinate deviation of various hole actual axis in the hole group, then calculate the specific numerical of hole set position error, But it is only suitable for two simple cases that is the hole group theoretical frame is fixed and the translational hole group theoretical frame.

Because the position tolerance zone annotation has many advantages, it become a more reasonable marking method for the limiting holes group center distance change, so the error evaluation which can be to adapt has become the domestic and foreign scholars pay much attention to the problem [4-5].

\section{Geometric Frame Optimization Method}

The method of location tolerance marked by compound location tolerance that is the most representative in uniformly distributing hole set position tolerance marking, other tagging error method are special cases in composite position error method.

Figure 1 is the label example of uniformly distributing of the circle uniform hole. It means the request for the hole group to axis $\mathrm{A}$ and axis $\mathrm{B}$ degrees of tolerance. Because ideal axis of distribution round are coincide with the datum axis and the distribution of diameter use the dimension theory, which make the ideal axis of each hole to be fixed. It means theory of geometrical frame is fixed. 
The tolerance zone is shown in figure 2(1). The position error method is the same as the method for group hole in rectangular located hole group according to the benchmark position error. The following frame represents further requirement for the hole group of each hole position tolerance, It is only require that the datum A keep vertical and has nothing with datum B so the frame theory can be rotated. Because of the hole group of the datum B satisfy the maximum principle, the scope of activities for reference axis can be compensated when the benchmark deviates from the maximum material size [6-9].

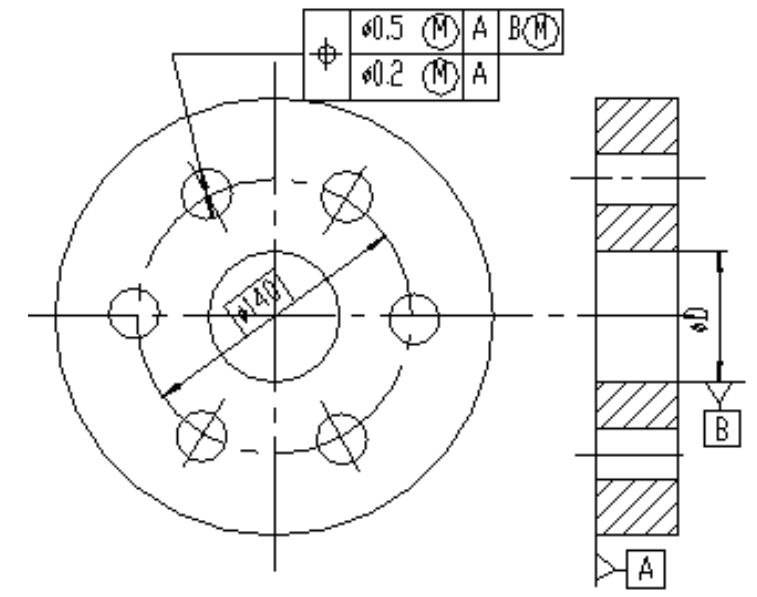

Fig. 1 Circumference hypodispersion hole's positional error dimensioning

Because the ideal axis distribution round the axis coincide with datum axis, the axis theory of each hole can deviate and the change way like a rigid body (That means the vertical A must in the circumference which the diameter is 140 and the included angle is $60^{\circ}$ ), so the axis of each hole and the theory frame of hole group can be moved in the reference hole tolerance compensation circumference. The tolerance zone diagram are shown in figure2(2). Then the method for position errors of hole in each group is the very complex. We must use the optimization frame method.

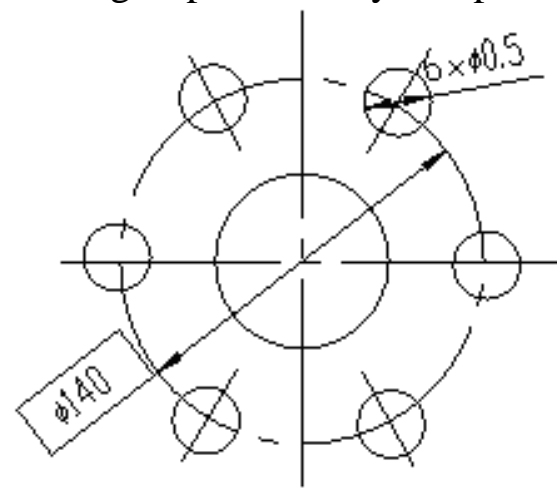

(1)

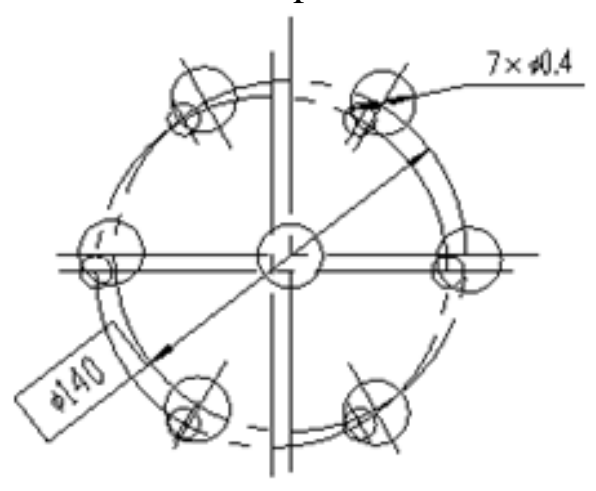

(2)

Fig. 2 Tolerance zone figure

The geometric frame optimization method is marking the coordinate deviation of each hole axis in the band diagram registration tolerance, and then get the point which called the hole measured point, then marking the rotation vector graph of the corresponding center of theoretical hole group in the hole measured point(it embodies the rotation of hole group frame theory).According the principle and method, we can use graphic method calculate minimum contain circle which the theory frame of hole group by rotation or translation after reaching the optimal position, which means got the uncertainty in position [7-10]. Geometric frame optimization principle is intuitive and image frame, it can not only draw the diagram of coincidence tolerance zone which means solving process diagram can also draw the intuitive diagram of hole group geometry frame translation or rotation. This provides message about the cause of error analysis or the repaired frock design or improved processing methods.

Translational Hole Group Theory Frame. After translational hole group theory frame 
calculated smaller contain circle under some condition, we should judge each theory center whether in axis hole tolerance compensation circle, If the tolerance compensation in tolerance compensation circle, the smaller contain circle is correct, otherwise it is invalid.

Rotational Hole Group Theory Frame. A smaller contain circle is obtained under the translational hole group theory frame, because the hole group theory frame can be rotated, this contain circle may further narrowing under new rotational conditions, and the rotational hole group theory frame come to a realization with the help of the rotating vector diagram of tolerance zone frame [11-12].

1) Determine the rotational center of theoretical frame

The corresponding rotating vector diagram be based on each points on a contain circle in registration tolerance frame. Then identify whether a rotating center make points on contain circle contract to circle, and it is required.

After rotating center is solved, moving direction and the ratio between of each actual point are shown in the rotating vector diagram of each actual point when the frame of rotating hole group is base on the rotating center mentioned before. Find the new points and the smaller containing circle.

2)The discriminant condition of termination error circle

Because the uniformly distributing hole group is relate to datum hole $B$, rotation and translation of theory frame shall not exceed the compensation tolerance circle of datum hole B.It called finally error which get last is a restricted termination error circle.

\section{The Establishment of Mathematical Model}

From the above, we know the process of the geometric frame optimization method is very complex, so it is necessary to establish the corresponding mathematical model if we want to finish it with the help of the computer [13-14].

Judging the Mathematical Model of Rotating Center that Make Contain Circle Shrunk. We only need consider the point on circle to judge whether there is a rotation center makes inclusive circle to shrunk, so the angle between each vector of point in circle and tangent is $M_{I}(i, 5)=1$, Among them $I=1,2 L L N, i=1,2 L L 2 N$.

Searching a Mathematical Model under Rotations Around the Center of Circle. The measured point rotate in the opposite direction when the theory frame rotating clockwise and counterclockwise in the same center of rotation, if the center is $M=1, B_{1}=B_{0}-N$, when the theory frame rotating counterclockwise, $M=1, B_{1}=B_{0}-N$,if the center is $B_{0}=1,2 L L N$ when the theory frame rotating clockwise, $M=2, B_{1}=B_{0}$.

$$
\text { So: } x(I)=x(I)+(-1)^{m} \times \sin L \times T
$$

$y(I)=y(I)+(-1)^{m} \times \cos L \times T$

Judging Geometric Frame Translation or Rotation Whether Beyond the Mathematical Model of Datum Hole Compensation Tolerance Circle.

$\left.B_{0}=i, i=2 N+1,2 N=2\right\}$,it means the center of datum hole is base on rotating center.

$x_{00}=x_{0}, y_{00}=y_{0}$

(3)

$$
\text { So: } S=\sqrt{x_{00}^{2}+y_{00}^{2}}+|F(1)-y(1)|
$$

The Size of Reference Hole Is a Mathematical Formula of Maximum Entity. The search formula is the same as a circumferential uniform distributing hole group $B_{0}=2 N+1,2 N+2$ with the above when the rotation center is $2 N+1$ or $2 N+2$.

\section{Example of Six Hole Group Uniform Circle and Benchmark Unlimited Compensation}

Program is running! 
Please input the parameter:

$$
\begin{aligned}
& \mathrm{N}=6 \\
& \mathrm{C} 0=1 \\
& \mathrm{~T}=10 \\
& \mathrm{R}=9999 \\
& \mathrm{X} \quad \mathrm{Y} \\
& 40,0 \\
& -21,35 \\
& 16,21 \\
& 5,40 \\
& 6,50 \\
& 34,1 \\
& X(1)=40 \quad Y(1)=0 \\
& X(2)=19.8108576 \quad Y(2)=35.686551 \\
& X(3)=10.1865765 \quad Y(3)=-24.3563885 \\
& X(4)=-4.9998394 \quad Y(4)=-40.0000133 \\
& X(5)=-46.3012002 \quad Y(5)=-19.8040113 \\
& \mathrm{X}(6)=16.1338422 \quad \mathrm{Y}(6)=29.9449350 \\
& \text { ***1st search } \\
& \mathrm{X} 0=-4.9875697 \quad \mathrm{Y} 0=-1.89692624 \\
& \mathrm{D} 0=90.0550890 \\
& \mathrm{X} 0=-7.21334964 \quad \mathrm{Y} 0=7.80249664 \\
& \text { D0 }=95.7074572 \\
& \mathrm{D}=90.055089 \quad \mathrm{~K} 0=3 \\
& \mathrm{X} 0=-4.9875697 \quad \mathrm{Y} 0=-1.89692624 \\
& 1 \quad 2 \quad 5 \\
& \text { B0 }=7 \quad \mathrm{~T}=10 \\
& \text { ***2nd search } \\
& \mathrm{X} 0=-0.913890274 \quad \mathrm{Y} 0=31.4312458 \\
& \mathrm{D} 0=103.1866200 \\
& \mathrm{X} 0=0.800514229 \quad \mathrm{Y} 0=3.72359755 \\
& \mathrm{D} 0=78.7518854 \\
& \mathrm{X} 0=2.50112129 \quad \mathrm{Y} 0=1.05239583 \\
& \mathrm{D} 0=81.1340098 \\
& \mathrm{D}=78.7518854 \quad \mathrm{~K} 0=10 \\
& \mathrm{X} 0=0.800514229 \quad \mathrm{Y} 0=3.72359755 \\
& 1 \quad 6 \quad 5 \\
& \mathrm{~B} 0=11 \quad \mathrm{~T}=10 \\
& * * * 3 \text { th search } \\
& \mathrm{X} 0=8.83977101 \quad \mathrm{Y} 0=71.2185575 \\
& \mathrm{D} 0=178.212089 \\
& \mathrm{X} 0=-1.67232929 \quad \mathrm{Y} 0=0.0991994031 \\
& \text { D0 }=72.6025289 \\
& \mathrm{X} 0=2.53517902 \quad \mathrm{Y} 0=-8.73123008 \\
& \mathrm{D} 0=80.7351921 \\
& \mathrm{D}=72.6025289 \quad \mathrm{~K} 0=10 \\
& \mathrm{X} 0=-1.67232929 \quad \mathrm{Y} 0=0.0991994031 \\
& 1 \quad 6 \quad 5 \\
& \mathrm{~B} 0=11 \quad \mathrm{~T}=10 \\
& * * * 4 \text { th search } \\
& \mathrm{X} 0=19.7084442 \quad \mathrm{Y} 0=47.6902097 \\
& \mathrm{D} 0=155.49398
\end{aligned}
$$



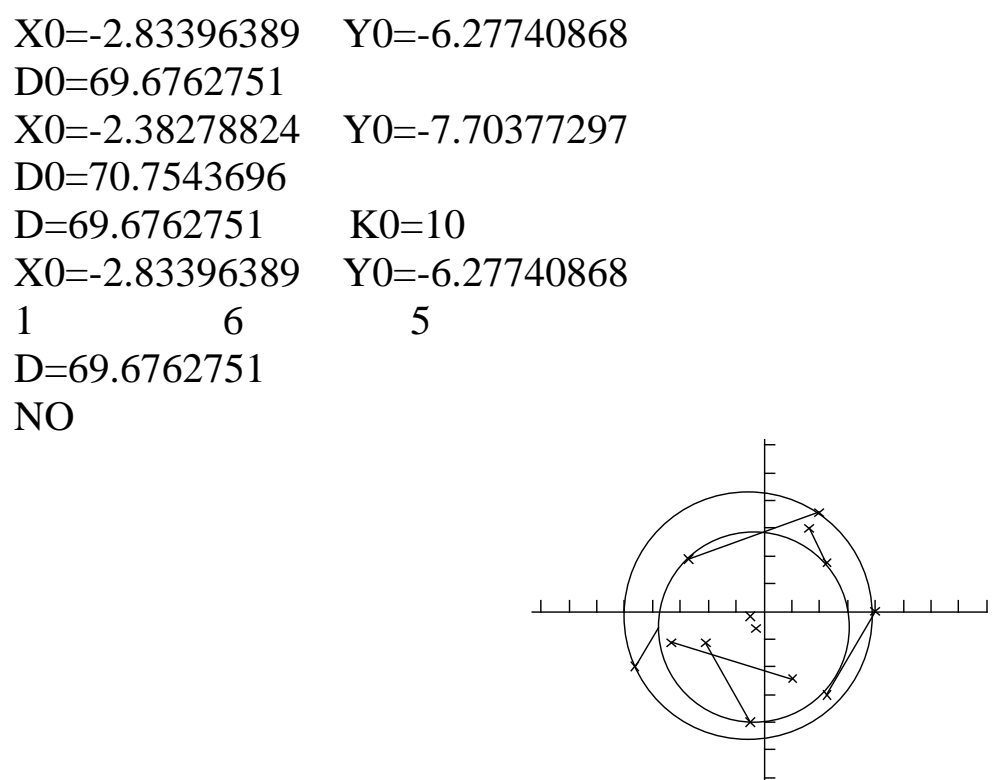

Fig. 3 Six hole group uniform circle

\section{Summary}

According to the principle of hole group position error and the establishment mathematical model above, compiling the $C$ language program which can solve the position error of hole group uniform circle, Finally, the measured data calculated by the actual parts ,the programming is considered practical and the mathematical model is corrected, as shown in figure3.

\section{Acknowledgement}

This work was supported by Dalian Vocational \& Technical College, we are indebted to the support and encouragements received from the staff and colleagues of the school of computer engineering.

\section{References}

[1] Yan yan Wu, Jami J.Shah, Joseph K.Davidson. Computer Modeling of Geometric Variations in Mechanical Parts and Assemblies. Transactions of the ASME Vol.3 ,MARCH 2003:54-63.

[2] Wei dong Wu, S. S. Rao. Interval Approach for the Modeling of Tolerances and Clearances in Mechanism Analysis. Journal of Mechanical Design JULY 2004,Vol,1 26:581-592.

[3] Zou Z, Morse EP. A gap-based approach to capture fitting conditions for mechanical assembly. Computer Aided Design 2004; 8:69 1700.

[4] Wittwer J W, Chase K W, Howell L L. The direct linearization method applied to position error in inematic linkages. Mech Mach Theory 2004; 81-93.

[5] Irfan Anjum Manarvi, Neat P.Juster. Framework of an integrated tolerance synthesis Model and using FE simulation as a virtual tool for tolerance allocation in assembly design. I.A . Journal of Materials Processing Technology 150 (2004):182-193

[6] MA Li-min, WANG Shu-zhen, JIANG Xiang-qian, Li Zhu, X Zhen-gao. Analysis of Geometrical Specification Model Based on the New Geometrical Product Specification Language. Journal of Shanghai University(English Edition), 2004. 8:7-11

[7] Hillyard R.C. Characterizing Non-Ideal Shapes in Terms of Dimensions and Tolerances Computer Graphics. 12(3). 234-238.

[8] Yan yan Wu, Jami J.Shah, Joseph K.Davidson. Computer Modeling of Geometric Variations in Mechanical Parts and Assemblies. Transactions of the ASME Vol.3 ,MARCH 2003:54-63. 
[9] Roy.U, Li, B. Representation and interpretation of geometric tolerances for polyhedral objects.1 1.:Size orientationand position tolerances. Computer-Aided Design. Vol 31 (4 ), 1999, 273-285

[10] Chen,M il-Chen. Tolerance synthesis by neural learning and nonlinear programming. International Journal of Production Economics, Vo 170(l), 2001,55-65

[11] G Moroni,W.Polini. Tolerance-based Variations in Solid Modeling. Journal of Computing and Information Science in Engineering. DECEMBER 2003,Vol.3:3 45-352.

[12] Wei dong Wu, S. S. Rao. Interval Approach for the Modeling of Tolerances and Clearances in Mechanism Analysis. Journal of Mechanical Design JULY 2004,Vol,1 26:581-592.

[13] Zou Z, Morse EP. A gap-based approach to capture fitting conditions for mechanical assembly. Computer Aided Design 2004; 8:69 1700

[14] Wittwer J W, Chase K W, Howell L L. The direct linearization method applied to position error in inematic linkages. Mech Mach Theory 2004; 81-93. 